\title{
Simulation Research of Manipulator Control System Based on Solidworks and Simmechanics
}

\author{
Yingjie Tang*, Xinwen Li \\ College of Nuclear Technology and Automation Engineering, Chengdu University of \\ Technology, Chengdu 610059, China \\ *Corresponding author e-mail: tngyjie@163.com
}

\begin{abstract}
Using Solidworks and Simulink simulation platform, the virtual simulation system of manipulator is built, which improves the design efficiency of manipulator control system. Firstly, the 3D model of the manipulator is established in Solidworks, and the industrial six degree of freedom manipulator is taken as the research object, the Solidworks model is introduced into Simulink, and the joint simulation control system of the manipulator is built by the SimMechanics module in MATLAB/SIMULINK, and the model of the manipulator is added in the motion simulation platform. The kinematic and dynamic analysis of the manipulator is carried out by adding the terminal pose signal and the joint signal (angle function and torque value).
\end{abstract}

KEYWORDS: SolidWorks, Robot, Matlab, Simulation, SimMech

\section{Introduction}

With the development of industral manipulator, industrial manipulaor has been widely used in various fields of industry. Manipulator is a complex mechanical and electrical system. The mechanical and electrical system of the manipulator designed needs to include driving force or torque driver. In designing the control system of the manipulator, we need to obtain the mathematical model of each part, calculate the required torque and rotation angle of each motor, and predict the position of the manipulator. In this paper, the six-degree-of-freedom industrial manipulator is taken as the research object, and the simulation research is carried out based on MATLAB /SimMechanics simulation plaform.

SimMechanics simulation platform is characterized by: no need to establish the mathematical model of mechanism movement and complex program; system modeling is convenient and intuitive, simulation function is powerful; system parameters can be optimized by changing the structure, and analysis results in the 
simulation software environment. These characteristics greatly reduce the workload of establishing the mechanism motion simulation model. When we study the manipulator, we can obtain the model of the dynamic system of the manipulator by this simple and fast method. SimMechanics includes a library of modules and special simulation interfaces (sensors and drivers) that connect the SimMechanics module to the Simulink environment. The joint angle signal is added to the joints of the simulation model, and the kinematics analysis of the manipulator is carried out.

\section{SimMechanics modeling technique}

SimMechanics can simulate the motion of the mechanism and provide a series of tools to solve the motion problems of the mechanism system with static constraints, coordinate transformation, etc. It can display the animation schematic diagram of the mechanism system motion with the functions provided by the virtual reality toolbox. In the mechanism model of Sim Mechanics, the special Sensor module and Actuator module can be connected with the general Simulink module. Using the basic concept of Newton dynamics, the rigid body connected by various motion pairs can be modeled and simulated to realize the purpose of analyzing and designing the mechanism system.

\section{Construction of simulation platform based on Matlab and Solidworks}

In the design of mechanical system, the three-dimensional solid model often built by the powerful modeling function of Solidworks software, which is then converted into XML format and imported into MATLAB for finishing. Simulink software package is used to model, simulate and analyze the dynamic system in advance to simulate the dynamic system in order to form the actual mechanism. It is necessary to make timely amendments to reduce the time of system modification and achieve the goal of efficient development.

In order to realize the construction of simulation platform based on Matlab and Solidworks, It is necessary to install Simscape Multibody Link plug-in unit in Solidworks software to establish Matlab simulation interface. The steps of building the simulation platform are as follows:

(1) According to the performance parameters of the computer and the version of MATLAB, download the appropriate install addon. $m$ and smlink. r2010b. Win32 compressed files from the official website of Math Works.( here is the 2010b version.)

(2) According to the installation path of the smlink.r2010b.win32 compressed package, set the corresponding install addon. Then run the installation command in the workspace of MATLAB to complete the installation of Simmechanics link in MATLAB.

(3) In order to make Matlab an automatic server. Run on Matlab's workspace: regmatlabserver. 
(4) Install Solidworks plug-in unit: run the command in Matlab work's space: smlink_linksw;

(5) Create a three-dimensional model in Soldwork and check "Simscape Multibody Link" (Figure 1). After modeling and saving *.SLDASM, save it as.Xml format file.

(6) Three-dimensional Solidworks model: mechimport (XX. xml) is imported into MATLAB, and the modeling process of MATLAB can be completed by running this command. Simulink module is added to realize the control and simulation analysis of the mechanism.

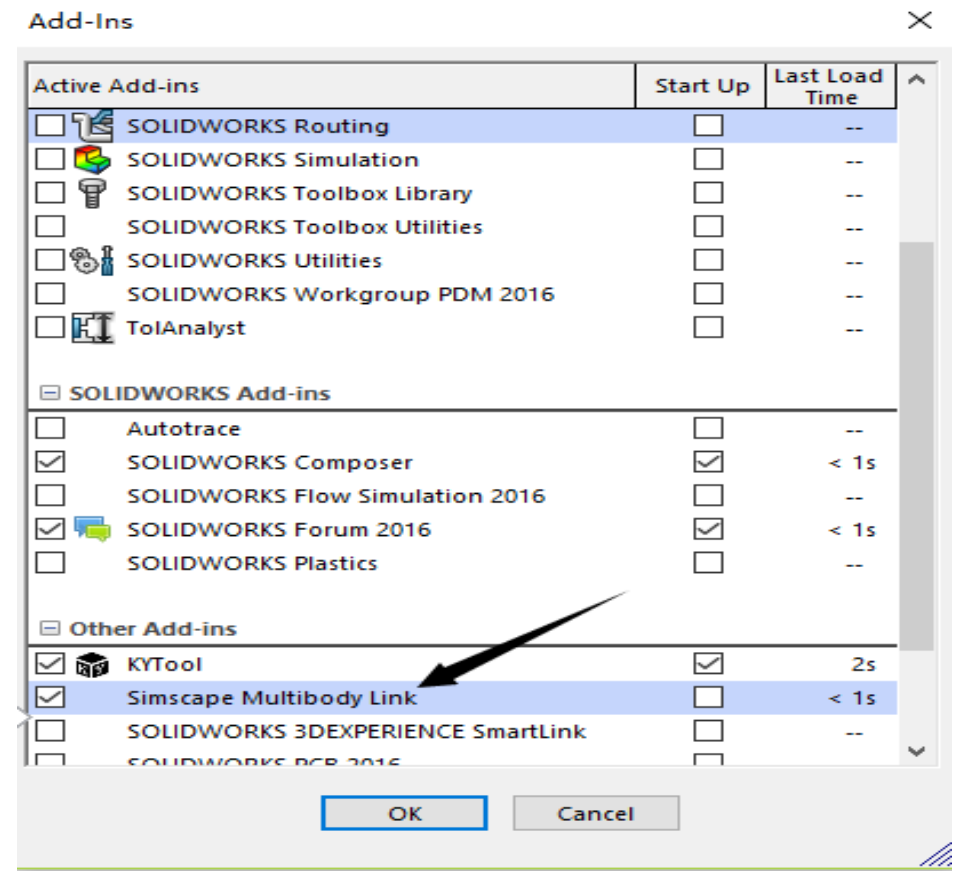

Figure. 1 Plug-in interface

\section{Establishment of simulation model}

The simulation process includes four steps: the establishment of mechanical system, the setting of model parameters, the establishment of MATLAB control model and the post-processing of results. In Soldworks, the SimMechanism plug-in unit is used to save the three-dimensional model of the four-axis palletizing robot as an "XML format" file (this file is only an intermediate transition file), and then run the mech_import ('Robot.xml') command in MATLAB to convert the XML file into an MDL file, which is called the SimMechanism model. The model transformation process is shown in Figure 2. 


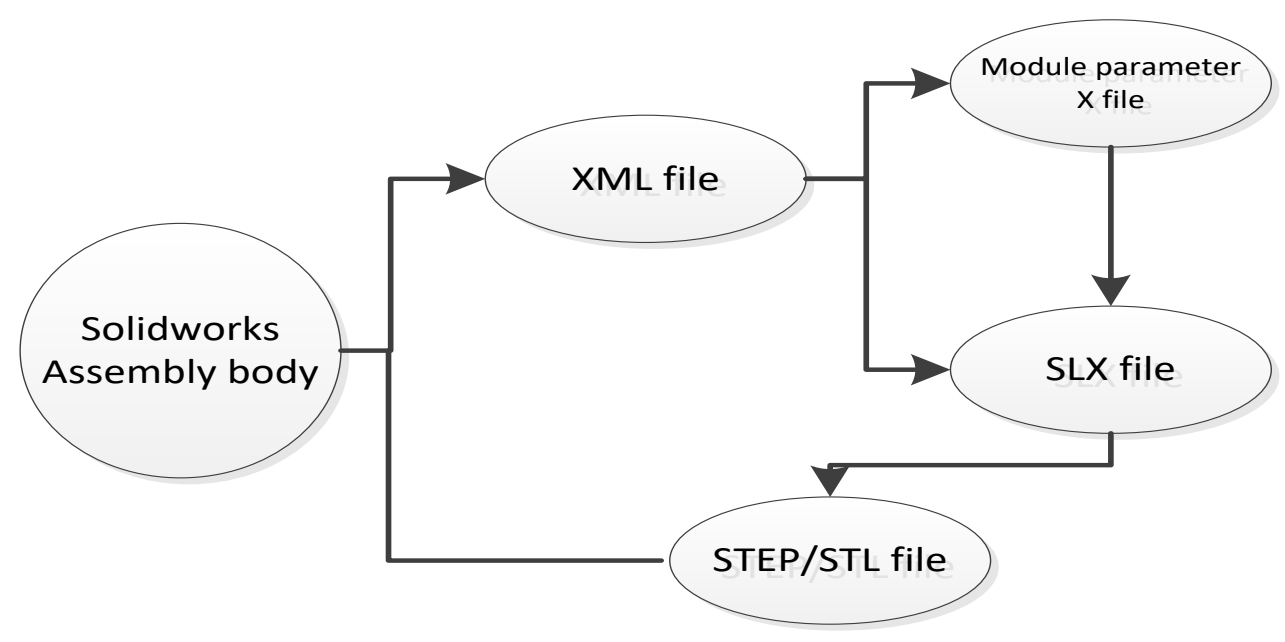

Figure. 2 Schematic diagram of model transformation process

This paper refers to a six-degree-of-freedom joint robot, mainly composed of the base, waist, arm, arm, wrist, including six rotating degrees of freedom are waist rotating joint, arm swing joint, arm swing joint, wrist rotating joint. The position of the end of the robot tool is achieved by controlling the joint rotation of the waist, arm and forearm. The attitude of the end of the robot tool is achieved by controlling the rotation of the waist and wrist joints. The wrist swing joint uses two parallel quadrilateral structures in series to keep the wrist perpendicular to the ground, thus reducing the control of one joint.

In order to facilitate the analysis of the following simulation module, it is necessary to simplify the design of the three-dimensional model. First, the assembly is partitioned by taking each joint as a node, and the components of each node are composed of its corresponding sub-assembly. Second, the coordination relationship among the sub-assembly and parts is reasonably selected to ensure the transformation of Sim-Mechanics model. The modules in the model are connected with the lowest pair (rotating pair R and moving pair P) to the greatest extent; (3) For the convenience of data and graphics processing, it is necessary to set up the relative position relationship between the world coordinate system and the assembly coordinate system reasonably; (4) neglect some small quality parts and simplify the model as far as possible. The simplified 3D model diagram is shown in Figure 3 below. 


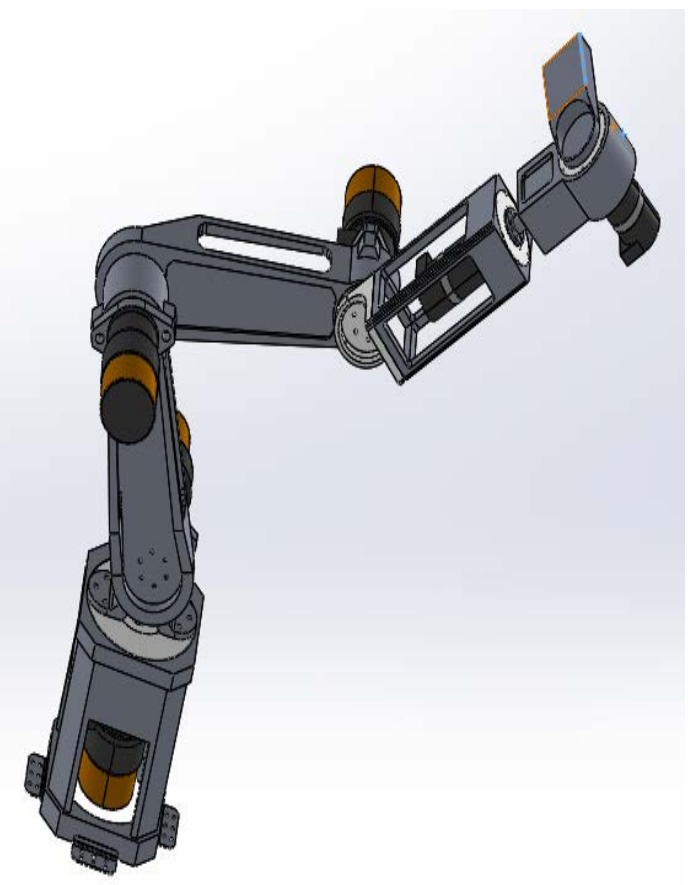

Figure. 3 3D model of robot

In the process of establishing the model of Solidworks mechanical system, we should select the appropriate parts, fix the origin of its coordinate system to the origin of the world coordinate system, and set the direction of $\mathrm{X}, \mathrm{Y}, \mathrm{Z}$ coordinate axes reasonably. The two steps of mechanical system establishment and model parameter setting are to export a module that can be used in MATLAB / Simulink / SimMechanism. This module contains the information parameters of the Solidworks model and has input and output interfaces, as shown in Figure 4.

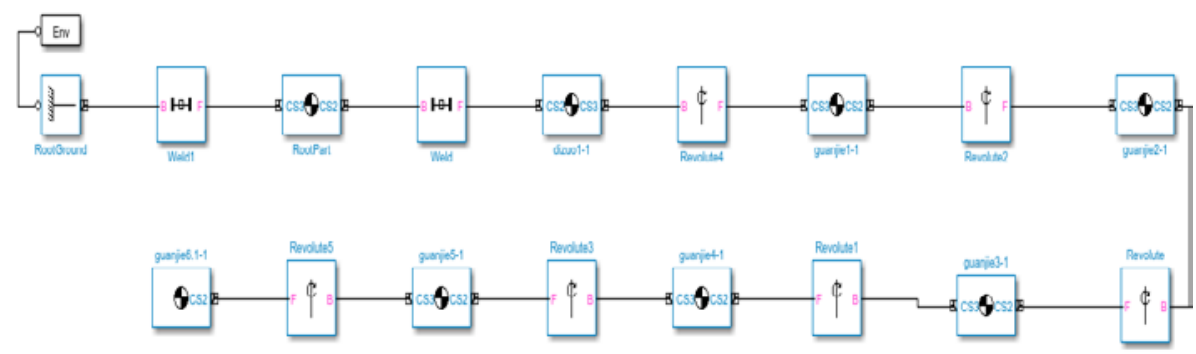

Figure. 4 Simulation model of manipulator 
Academic Journal of Computing \& Information Science ISSN 2616-5775 Vol. 1, Issue 1: 19-27, DOI: 10.25236/AJCIS.010003

The motion visualization model at runtime is shown in Figure 5.

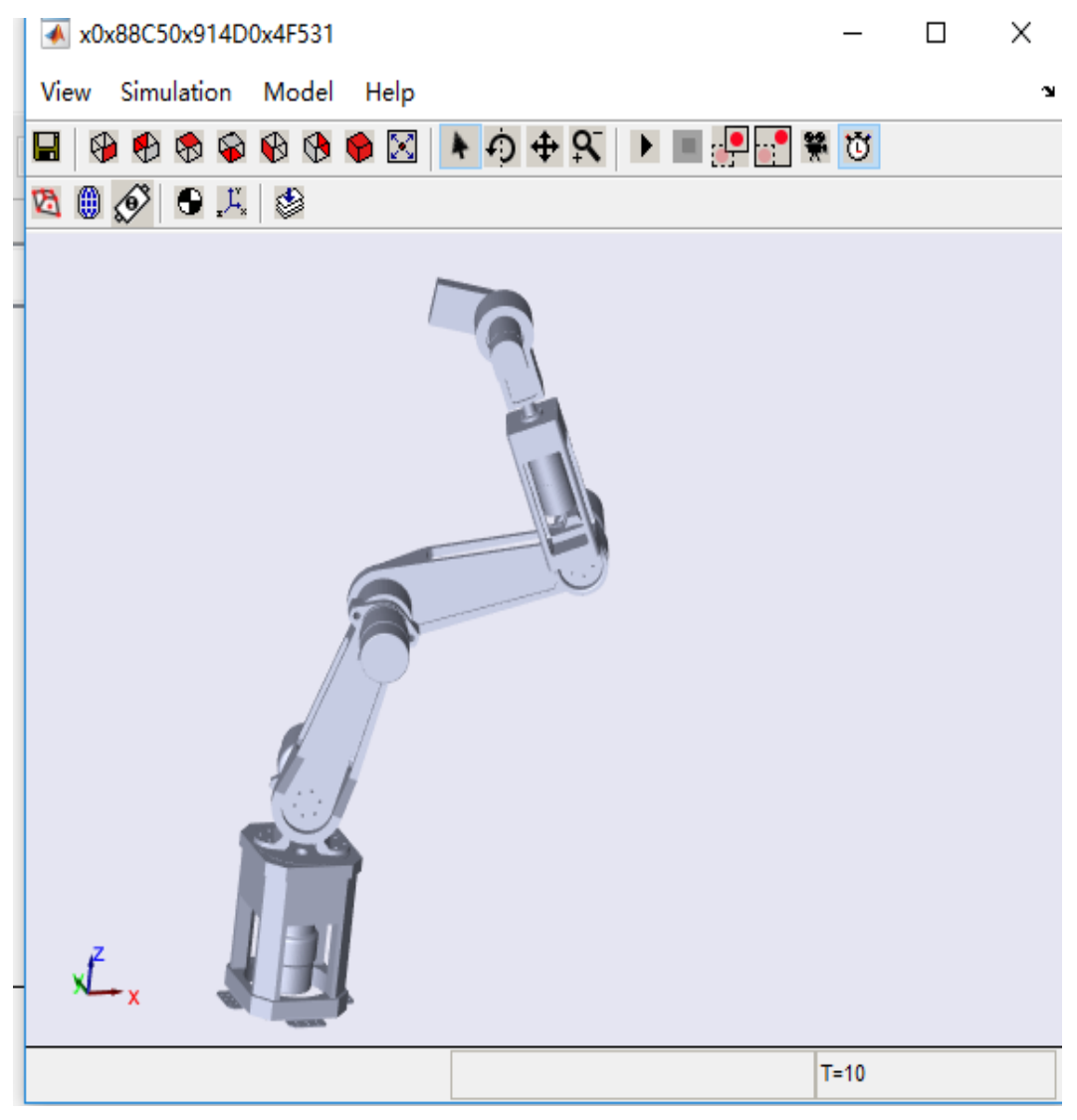

Figure. 5 Visualization model of manipulator

5. Kinematics and dynamics analysis of six degree of freedom manipulator

\subsection{Kinematics analysis of manipulator}

The joint angle driving signal is shown in Figure 6. 


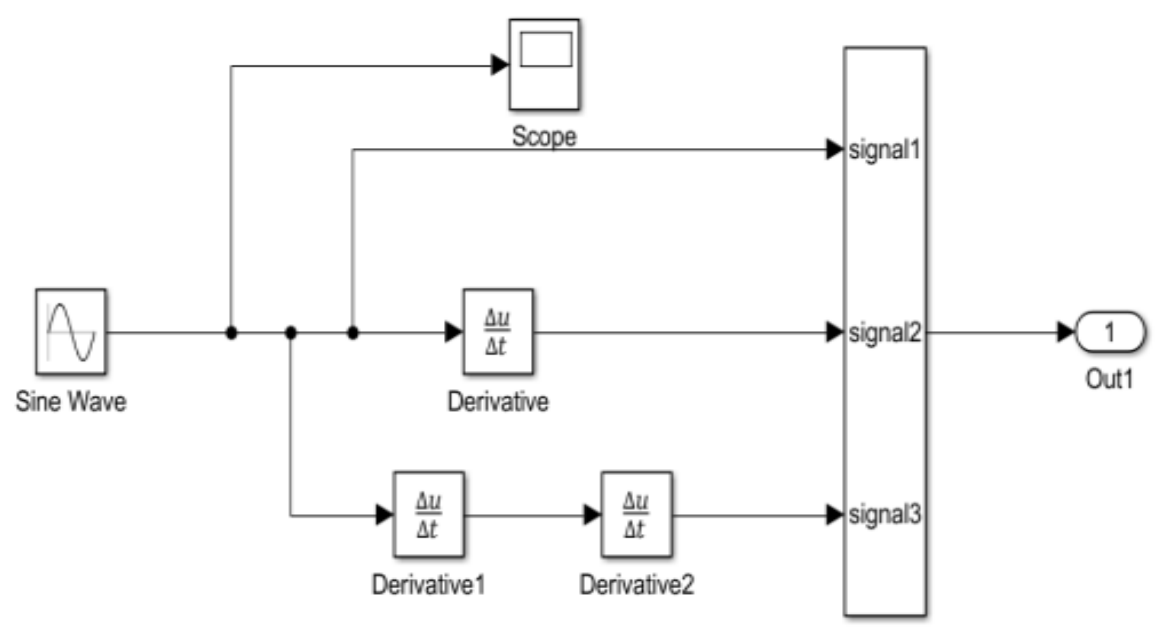

Figure. 6 Joint angle driving signal

The forward kinematics solution of the manipulator is based on the values of joint variables, such as theta_1, theta_2, theta_3, theta_4, theta_5, theta_6. According to this requirement, in order to facilitate the analysis of kinematics forward solution, the variation values of each joint angle are obtained. Joint 1 angle change function is theta_1=35sint=; joint 2 angle change function is theta_2=20sin (t-pi/2); joint 3 angle change function is theta_3=10sint; joint 4 angle change function is theta_ $4=5 \sin (\mathrm{t}-\mathrm{ps} / 2)$; joint 5 angle change function is theta_ $5=15 \sin (\mathrm{t}-$ pi/2); joint 6 angle change function is theta_6 $=25$ sint. The angular velocity and angular acceleration function can be obtained through derivation of the angle functions of the joints. The model of the six degree of freedom manipulator with the joint angle driving signal is shown in Figure 7.

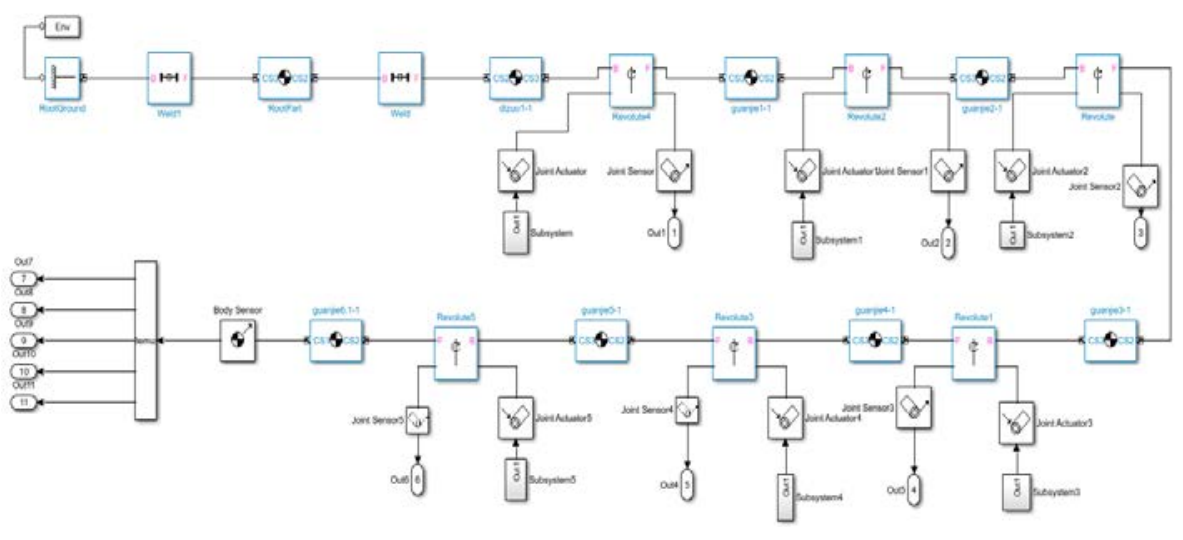

Figure. 7 SimMechanics system model 
The change curves of each joint angle function are shown in figures 8 .
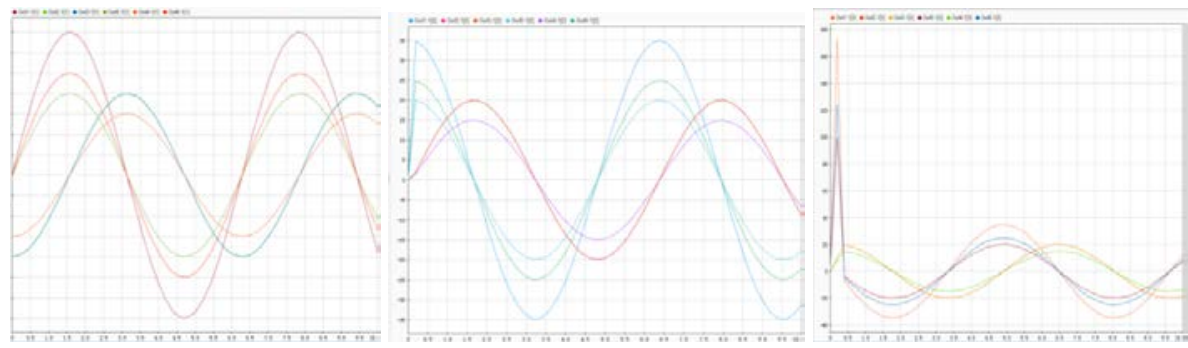

Figure. 8 Change of joint angular velocity, angular and acceleration

\subsection{Dynamics analysis of manipulator}

According to the joint driving force or torque, the movement of the robot arm (joint displacement, speed and acceleration). Now add the driving torque $\mathrm{M}=10 \mathrm{~N} /$ $\mathrm{m}$ on the first three rotating joints, and add the driving torque $\mathrm{M}=3 \mathrm{~N} / \mathrm{m}$ on the last three rotating joints.

Add the drive signal and output module, set the Joint Sensor module in the SimMechanics module, and select the output as the joint moment. The torque variation curve of the manipulator after simulation is shown in Figure 9.

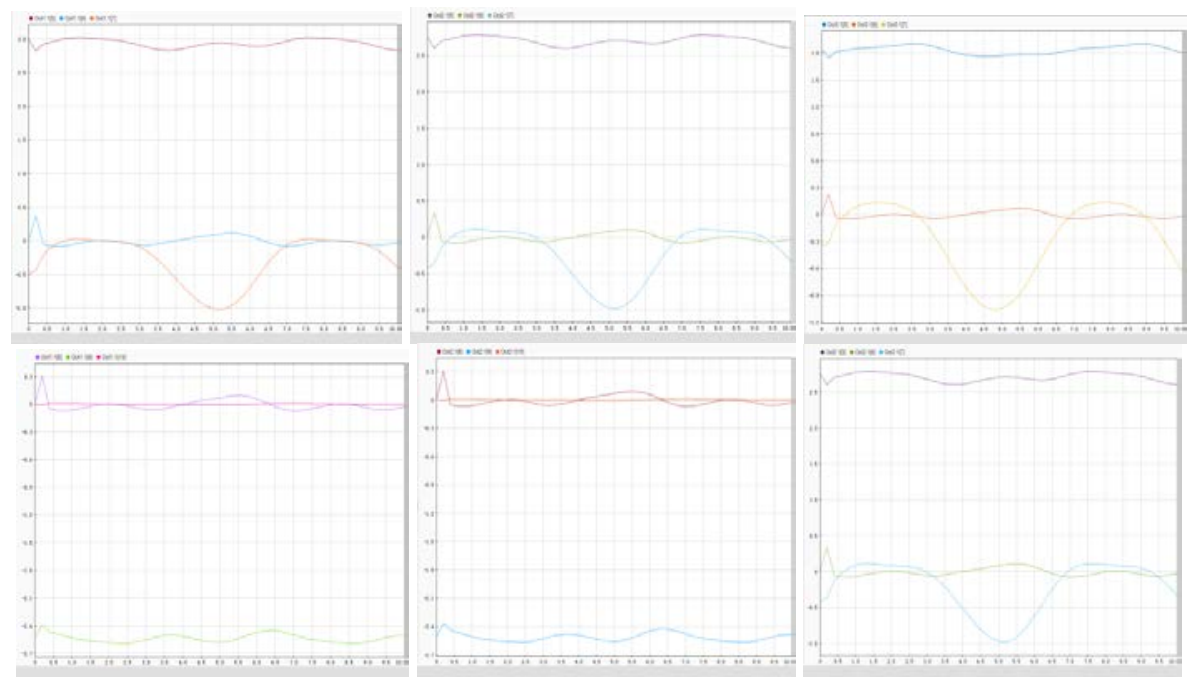

Figure. 9 Torque of each joint 
In the actual operation process, the influence of joint angle change on joint torque can be analyzed by the change of dynamic inverse solution of the manipulato.

\section{Conclusion}

The kinematics and dynamics simulation of the six DOF Manipulator is studied and analyzed. The research and design of the relevant control of the manipulator are based on kinematics and dynamics. The three-dimensional model is established by Solidworks and imported into the MATLAB/SimMechanics simulation platform. The kinematics and dynamics of the robot are analyzed. The workload of establishing the mechanism simulation model is greatly reduced and the manipulator movement is analyzed. The change of joint angle and posture in forward and inverse kinematics, and the change of angle and torque in forward and inverse kinematics are interacted with each other to prepare for further control design of manipulator.

\section{References}

[1] H.P. Zhang, Y.H. Wang, H.Q. Chen (2017). Kinematics analysis and Simulation of six degree of freedom manipulator. Industrial instrumentation and automation devices, vol.16, no.4, p.127-130.

[2] Y.P. Lv (2017). Rigid flexible coupling dynamics of three degree of freedom 3RPS parallel manipulator. Lanzhou University of Technology.

[3] Y.J. Yang, X.L. Chen (2017). Kinematic analysis of a universal six degree of freedom industrial robot. Scientific and technological information, vol.15, no.3, p.97-99+101.

[4] X. Li (2017). Research on the development and trajectory planning method of six degree of freedom articulated robot system. East China University of Science and Technology.

[5] Z. Ye (2017). Research and Simulation of trajectory planning algorithm for six degree of freedom industrial robot. Nanjing University of Aeronautics \& Astronautics.

[6] M.T. Wang, L.A. Zhang (2017). Kinematic analysis of six degree of freedom robot. Journal of Anhui University of Technology (NATURAL SCIENCE EDITION), vol.33, no.4, p.365-371.

[7] T. Song, L.J. Wang, L.P. Wang (2017). Kinematics simulation analysis of four degree of freedom serial robot. Coal mine machinery, vol.38, no.1, p. 43-45.

[8] M.X. Shi (2017). Kinematics modeling and Simulation of 3-PRRRR parallel robot. Tianjin University of Technology.

[9] Y. Gao, G.Q. Ma, Z.H. Yu, G.H. Zao (2016). Kinematics analysis and 3D visualization simulation of an industrial robot with six degrees of freedom. China Mechanical Engineering, vol.27, no.13, p. 1726-1731.

[10] R.P. Ye (2016). Kinematic modeling and Simulation of five DOF serial manipulator. Shenzhen University.

\section{Published by Francis Academic Press, UK}

